\title{
Valle de la muerte: factores que dificultan el éxito de innovaciones tecnológicas *
}

\author{
Valley of Death: Factors Hindering the Success of Technological Innovations
}

\author{
Elizabeth Jiménez-Medina (iD) \\ Magíster en Gestión Tecnológica, Institución Universitaria Pascual Bravo, \\ Medellín - Colombia, e.jimenezme@pascualbravo.edu.co \\ René Yepes-Callejas \\ Magíster en Gestión Tecnológica, Universidad Pontificia Bolivariana, \\ Medellín - Colombia, edgar.yepesc@upb.edu.co
}

\begin{abstract}
Jim Giraldo-Builes iD
Magíster en Gestión de Ciencia, Tecnología e Innovación, Institución Universitaria Pascual Bravo, Medellín - Colombia,jim.giraldo@pascualbravo.edu.co
\end{abstract}

\begin{abstract}
Iván Darío Rojas-Arenas
Magister en Gestión de la Innovación Tecnológica, Cooperación y Desarrollo Regional, Institución Universitaria Pascual Bravo, Medellín - Colombia, ivan.rojasar@pascualbravo.edu.co
\end{abstract}

Cómo citar / How to cite

Jiménez-Medina, E., Yepes-Callejas, R., Giraldo-Builes, J., Rojas-Arenas, I. D. (2021). Valle de la muerte: factores que dificultan el éxito de innovaciones tecnológicas. Revista CEA, v. 7, n. 15, e1926.

https://doi.org/10.22430/24223182.1926

Recibido: 12 de mayo de 2021

Aceptado: 24 de agosto de 2021

\section{Resumen}

El término valle de la muerte es una metáfora utilizada para describir una etapa de vacío o cuello de botella que experimentan los proyectos de innovación tecnológica después de las fases de investigación y desarrollo, y antes del desarrollo de nuevos productos y su lanzamiento al mercado. El objetivo de este artículo fue estudiar los factores involucrados en el valle de la muerte con el fin de ayudar en la definición de estrategias para superar esta brecha y, de este modo, aumentar las probabilidades de éxito de la innovación. Para ello se realizó una exploración de algunos de ellos y de relaciones causales a partir de entrevistas, así como de la revisión de información secundaria de

* Este artículo se deriva del proyecto titulado «Factores formadores del Valle de la Muerte de la I+D: casos comparativos SAPIENCIA vs otras instituciones de Medellín» y ha sido financiado por la Institución Universitaria Pascual Bravo. 
nueve intermediarios de innovación ubicados en Medellín (Colombia), encargados de gestionar el valle de la muerte para acelerar los proyectos de innovación tecnológica hasta las etapas previas a la explotación comercial. Entre los hallazgos más relevantes de la investigación se encontró que los intermediarios de innovación en empresas, universidades y programas de apoyo estaban de acuerdo en que los factores que más inciden en la aparición del valle de la muerte son la falta de competencias del talento humano/roles mal distribuidos, ceguera en las dimensiones y la debilidad en el trabajo en red. Finalmente, el estudio concluye que el análisis de los factores generadores del valle de la muerte requiere de una visión sistémica y compleja, de forma que se puedan tener en cuenta las distintas interacciones que se establecen entre ellos.

Palabras clave: Valle de la Muerte, innovación tecnológica, competencia de gestión, gestión de grupos de interés.

Clasificación JEL: O31, O32, O33.

\title{
Highlights
}

- La falta de competencias, la ceguera dimensional y el trabajo en red son determinantes del valle de la muerte.

- Falta de competencias del talento humano, ceguera dimensional y trabajo en red son los factores más relevantes para que un proyecto de innovación caiga en el valle de la muerte.

- Un factor de éxito en proyectos de investigación, desarrollo e innovación (I+D+i) es el desarrollo de competencias de gestión.

- Modelos mentales restrictivos generan dificultades para el trabajo en red.

- Las instituciones de educación superior (IES) públicas se ven afectadas para establecer alianzas con terceros debido a la normatividad vigente y la lentitud en los procedimientos.

\begin{abstract}
The term Valley of Death (VoD) is a metaphor used to describe a gap or bottleneck that technological innovation projects undergo after the research and development (R\&D) stage and before the development of new products and their market launch. This study analyzes the factors involved in the $\mathrm{VoD}$ to help define strategies to overcome this situation, thus increasing the chances of success in innovation. For this purpose, interviews were conducted to explore some of its factors and causal relationships. Additionally, this paper reviews secondary information provided by nine innovation intermediaries located in Medellín (Colombia) that manage the VoD to accelerate technological innovation projects prior to the stage of commercial exploitation. One of the most relevant findings is that innovation intermediaries at companies, universities, and support programs agree that the most determining factors for the emergence of the VoD are lack of competences of human resources/poorly distributed roles, dimensional blindness failure, and weak networking. In conclusion, analyzing the factors that lead to the $\mathrm{VoD}$ requires a systemic and complex vision that considers different interactions between them.
\end{abstract}

Keywords: Valley of Death, technological innovation, management competency, stakeholder management.

JEL classification: O31, O32, O33. 


\section{Highlights}

- Lack of competences, dimensional blindness failure, and networking are determinants of the Valley of Death.

- Lack of competences of human resources, dimensional blindness failure, and weak networking are the most influential factors that determine if a project falls into the Valley of Death.

- Developing managerial competencies is a success factor of Research, Development, and Innovation $(\mathrm{R}+\mathrm{D}+\mathrm{i})$ projects.

- Restrictive mental models hinder networking.

- Public higher education institutions are affected when they establish partnerships with third parties due to current regulations and slow procedures.

\section{INTRODUCCIÓN}

Aunque la innovación contribuye al mejoramiento económico y el éxito empresarial (Vaníčková y Szczepańska-Woszczyna, 2020; Ivanova et al., 2019), esta actividad suele ser percibida como compleja y con altas tasas de fallo por constituir un proceso impredecible, idiosincrásico y de larga duración (Hsu et al., 2014). En el caso de las innovaciones tecnológicas, que según García y Calantone (2003) y García Núñez et al. (2019), materializan invenciones desarrolladas por la actividad científica, la ingeniería y disciplinas industriales, es usual que dicha materialización complete diversas fases del proceso de investigación, desarrollo e innovación ( $1+D+i)$. En este sentido se ha acuñado la metáfora valle de la muerte (VM) para referir un estado problemático que restringe el avance de proyectos de innovación tecnológica (Andronchev et al., 2020), y que en últimas constituye una causa de fallo de las innovaciones (Auerswald y Branscomb, 2003, de Frutos Belizón et al., 2018).

Frank et al. (1996) y Alunni (2020) describen el VM como una situación de fallo que ocurre cuando los innovadores no pueden acceder a financiación para el escalamiento y fabricación de un producto resultado de un proceso de I+D. Para Auerswald y Branscomb (2003), el VM constituye una brecha persistente para la innovación, que involucra restricciones financieras, falta de articulación entre técnicos y especialistas en negocios, deficiencias en infraestructura y divergencias entre intereses investigativos y comerciales. Markham et al. (2010), plantean el VM como un corredor entre investigación y desarrollo (I+D) y una fase final de desarrollo de nuevos productos donde se presentan restricciones de capacidades.

Si bien se reconoce que el VM involucra múltiples factores (Auerswald y Branscomb, 2003; Markham et al., 2010), son usuales los análisis en relación con un factor específico o un grupo limitado de estos, con predominio del financiero (Frank et al., 1996; Beard et al., 2009; Auerswald y Branscomb, 2003; Munari et al., 2018), constituyendo, de esta forma, una línea explicativa dominante. Otras fuentes revisan factores no financieros como el entorno, instituciones y redes (Raven y Geels, 2010; Lai et al., 2012; Weiss y Bonvillian, 2013), competencias y roles del personal involucrado en las innovaciones (Markham, 2016; Markham et al., 2010), infraestructura técnica (Tassey, 2014) y actividades de marketing (Schoonmaker et al., 2013). La diversidad de enfoques sugiere un fenómeno complejo y multifactorial, propicio a un análisis sistémico que identifique relaciones causales entre factores; sin embargo, los trabajos previos han profundizado en algunos más específicos, más que en las 
relaciones entre ellos. En la Tabla 1 se muestran algunas definiciones del concepto de VM, dadas por autores relevantes dentro de esta temática.

Tabla 1. Algunas definiciones del VM

Table 1. Some definitions of the VoD

\begin{tabular}{lc}
\hline \multicolumn{1}{c}{ Definición valle de la muerte } & Autor (Año) \\
\hline $\begin{array}{l}\text { Brecha entre los proyectos de innovación y los } \\
\text { prototipos que de ella emergen, y la salida exitosa } \\
\text { de dichos prototipos al mercado (aplicación en } \\
\text { masa). }\end{array}$ & Merrifield (1995) \\
\hline $\begin{array}{l}\text { Brecha de recursos entre laboratorios o unidades } \\
\text { de I+D y la comercialización de la innovación. }\end{array}$ & Markham (2016) \\
\hline $\begin{array}{l}\text { Concepto utilizado para explicar la situación en la } \\
\text { que una tecnología no llega al mercado debido a la } \\
\text { incapacidad de avanzar en su demostración hasta la } \\
\text { fase de comercialización. }\end{array}$ & Auerswald y Branscomb (2003) \\
\hline $\begin{array}{l}\text { Lugar donde los buenos descubrimientos, } \\
\text { normalmente de laboratorio, van a morir porque } \\
\text { carecen de financiación para convertirse en un } \\
\text { producto comercial. }\end{array}$ & Beard et al. (2009) \\
\hline $\begin{array}{l}\text { Espacio entre la oportunidad descubierta y el } \\
\text { desarrollo del producto. }\end{array}$ & Markham et al. (2010) \\
\hline $\begin{array}{l}\text { Brechas entre la investigación básica y el desarrollo } \\
\text { de productos. }\end{array}$ & Gou et al. (2013) \\
\hline $\begin{array}{l}\text { Etapa que va desde que un emprendedor inicia su } \\
\text { proyecto y empieza a realizar gastos, hasta que } \\
\text { eventualmente logra que su empresa esté en } \\
\text { equilibrio, es decir, hasta que consigue al menos } \\
\text { tener ingresos iguales a sus gastos. }\end{array}$ & \\
\hline
\end{tabular}

Se debe entender que la complejidad de este fenómeno no se debe exclusivamente al número de variables que intervienen, sino también a la cantidad y calidad de relaciones que se establecen entre las mismas, lo cual presupone que un análisis lineal sería simplista o reduccionista y, por tanto, no daría una visión real de las dinámicas de dichas relaciones y los resultados que generan en proyectos de I+D+i. Así las cosas, si lo que se plantea es un análisis causa y efecto, mientras mayor sea la cantidad de factores analizados, más precisa será la explicación que se presente al VM como objeto de estudio, de allí que, basados en trabajos investigativos previos, se tengan en cuenta todos aquellos que se encuentran validados por la revisión bibliográfica para, posteriormente, escoger los más relevantes.

En este sentido, Jiménez (2017) realiza una investigación en la cual se buscaba determinar los factores más relevantes en la generación del VM en la ciudad de Medellín que, para tal efecto, y mediante el estudio de casos, plantea una evaluación de aquellos encontrados en la literatura y los que se identificaron por medio de encuestas realizadas a doce intermediarios de innovación de la ciudad, los cuales se encargan de gestionar el paso de los proyectos de I+D hacia la fase final de innovación; dicho estudio arrojo 33 factores (Tabla 2) que inciden en el VM en Medellín. 
Tabla 2. Barreras que inciden en el VM

Table 2. Barriers that influence the VoD

\section{Barreras que inciden en el Valle de la Muerte}

1. Falta de competencias del talento humano/Roles mal distribuidos.

2. Desconocimiento de las dimensiones.

3. Debilidad en el trabajo en red.

4.Falta de financiación y mala asignación de recursos.

5. Fallas institucionales (normatividad) para la innovación.

6.Modelos mentales incompletos o restrictivos.

7. Aversión al riesgo.

8. Lenta salida al mercado.

9. Falta claridad en el modelo de negocio.

10. Valores y creencias conservadores frente a las innovaciones.

11. Falta de apoyo del Gobierno.

12. Visión de corto plazo.

13. Falta de apoyos en sistema CTi (integración de telefonía informática).

14. Resistencia del cliente a adoptar.

15.Poca o inexistente infraestructura para escalamiento.

16. Confusión entre innovación incremental y radical.
17. Deficiencia de políticas internas.

18. Falta de soporte en la estructura organizacional.

19. Desconocimiento del proceso de alistamiento de una tecnología.

20. Poca comunicación con inversionistas.

21. Dificultad para acceder a canales comerciales.

22. Problemas de negociación y respaldo de la entidad matriz.

23. Falta de madurez de la tecnología.

24. Rigidez en la gestión de la innovación.

25. Débil convicción frente a la innovación.

26. Dicotomía en el rol de la Universidad.

27. Poca estabilidad económica.

28. Rigidez de empresas, por éxitos previos.

29. Conflictos en la propiedad intelectual.

30. Problemas de estructuras de mercado.

31. Mal manejo de finanzas personales.

33. Baja tolerancia a la ambigüedad.

Fuente: elaboración propia a partir de (Jiménez, 2017).

El VM se inicia en el momento en que se requiere pasar de un prototipo o invento a una idea conceptual y funcional, de forma que se puedan determinar los costos y requerimientos técnicos para su producción (Jiménez, 2017). Asimismo, afirma que las barreras a la innovación funcionan como inhibidores o cuellos de botellas que impiden el desarrollo de estas, generando así una alta tasa de fracasos en proyectos de I+D; de allí la importancia del estudio para determinar posibles soluciones a esta problemática.

En la presente investigación se toma como referencia el estudio de Jiménez (2017) como base para establecer los posibles factores que dificultan el éxito de las innovaciones tecnológicas en la ciudad y, de esta manera, a partir de la calificación establecida por los entrevistados dentro del proceso de obtención de información primaria ${ }^{1}$, priorizar aquellas barreras con mayor relevancia. De esta manera, se tomaron los siguientes factores: No dominio de las dimensiones, Falta de competencias

\footnotetext{
${ }^{1}$ Este proceso se detalla en el apartado metodológico.
} 
Valle de la muerte: factores que dificultan el éxito de innovaciones tecnológicas

y roles, Debilidad en los relacionamientos y redes, Falta de financiación, Modelos mentales incompletos o inadecuados, Fallas institucionales y Falta de apoyo al sistema CTi.

\section{No dominio de las dimensiones}

Se constituye como barrera cuando el proceso de innovación se organiza y gestiona sin haber considerado todas las dimensiones del sistema, incurriendo en ceguera dimensional (Van Lancker et al., 2016). Lettner et al. (2020) señalan que es necesario identificar brechas y asimetrías en el proceso de innovación a lo largo de la cadena de valor del desarrollo de producto para lograr la difusión de la innovación. De igual forma es preciso tener en cuenta la valoración de las partes interesadas o stakeholders sobre los diferentes atributos de valor de una tecnología como costos, condiciones del proceso, seguridad en el suministro y beneficios ambientales, entre otros, los cuales no necesariamente son aspectos tenidos en cuenta durante etapas de I+D (Padyab et al., 2020). Gimeno et al. (2018) y Barron y Amorós (2020) hallaron fallas en aspectos como el conocimiento del mercado y sus necesidades, falta de conocimiento de la industria en términos de regulaciones o requerimientos para comercializar en un mercado, competidores y la factibilidad de la tecnología. Estos aspectos se configuran de manera tal que se convierten en una barrera para la salida de innovaciones del laboratorio a la industria. Nemet et al. (2018) enfatizan en otros aspectos, como la motivación, la sincronización y el escalado, el rol del sector público y el de los mercados como necesarios para la demostración efectiva de una tecnología.

\section{Falta de competencias y roles}

Esta barrera se refiere al avance de competencias de talento humano que desarrolla los proyectos de I+D, como modelos de negocio y modelos financieros, entre otros, y a la distribución de los diferentes roles que hacen o deben hacer parte de los equipos (Markham, 2016; Markham et al., 2010). Por su parte, Sandberg y Aarikka-Stenroos (2014) identifican la falta de competencias en el descubrimiento, incubación, aceleración y comercialización como una barrera interna que afecta los procesos de innovación. Abereijo (2015) y Meyer et al. (2011) plantean la importancia de inclusión de las habilidades en actividades empresariales que permitan disminuir las brechas de comercialización en temas como el desarrollo de propuestas de valor, la identificación de mercados y la creación de modelos de negocio como un componente importante dentro de la carrera como investigador, que les permita la oportuna identificación y explotación de tecnologías. Liening et al. (2018) y Botha y Morallane (2019) sugieren que las facetas de los recursos emprendedores comprenden habilidades del individuo, como el conocimiento profesional, el conocimiento en emprendimiento y la personalidad, junto con conocimientos por fuera del aspecto personal, como el contexto del mercado y las barreras legales.

\section{Debilidad en los relacionamientos y redes}

Esta barrera se presenta cuando las interacciones son inexistentes o deficientes, y no permiten la formación de alianzas que conlleven a procesos de comercialización. (Rothaermel y Deeds, 2006). De esta manera, González Fernández et al. (2019) proponen que es necesario adelantar un relacionamiento entre los diferentes actores interesados como academia, industria y Estado que permitan superar el VM. Para esto, Markham y Mugge (2015) plantean modelos de trabajo colaborativo bajo innovación abierta para mejorar el relacionamiento. 


\section{Falta de financiación}

Este factor se evidencia cuando existen dificultades para acceder a recursos financieros o se presenta una distribución de recursos que no responde a otras necesidades diferentes de los procesos de investigación (Auerswald y Branscomb, 2003). Mientras tanto, Beard et al. (2009) sugieran que la falta de financiación es un factor determinante para la ocurrencia del valle de la muerte, y se constituye en una barrera para la generación de innovaciones. Daldrup et al. (2020), por ejemplo, afirman que estrategias como la microfinanciación pueden ayudar a la consecución de recursos financieros, así como a una mejor comunicación del conocimiento científico y una mejora de los procesos de transferencia tecnológica hacia la sociedad. Por otro lado, Gubitta et al. (2016) analizan el fenómeno que se presenta con las empresas derivadas de universidades, las cuales se enfrentan a serias dificultades para la financiación de sus proyectos debido, entre otras cosas, a la existencia de una marcada asimetría en la información.

\section{Modelos mentales incompletos o inadecuados}

Los modelos mentales se entienden como representaciones incompletas o erradas respecto a lo que se debe hacer para completar las innovaciones. Estos modelos conllevan a una mentalidad restrictiva (Vink et al., 2019; Lee-Kelley y Blackman, 2005). En esta línea, Surroca et al. (2014) desarrollan un estudio interesante en donde analizan grupos cognitivos presentes en empresas, llegando a la conclusión que se dan constructos comunes, los cuales funcionan como modelos mentales organizacionales que determinan el desempeño de la organización. Por su parte, Barron y Amorós (2020) plantean que el trabajo cooperativo entre entidades públicas y privadas permite cambiar la mentalidad muchas veces restrictiva de los investigadores, en la medida en que se creen puentes entre investigación y mercado, aprovechando sobre todo la experiencia que la empresa privada puede aportar.

\section{Fallas institucionales}

Fallas en normativas formales e informales, en relación con las innovaciones. (Negro et al., 2012; Van Lancker et al., 2016); lo anterior implica que dichas normativas dificulten o imposibiliten un adecuado relacionamiento entre agentes, así como el establecimiento de protocolos que en muchas ocasiones ralentizan el paso de productos de I+D a una fase de comercialización. Este tipo de factores parece más evidente en instituciones públicas, debido, según los autores, al exceso de burocracia y la poca eficiencia de los procesos.

\section{Falta de apoyo del sistema CTi}

Este factor se enfoca en la interacción de agentes del sistema CTi con los proyectos de I+D+i, dado que a partir de la misma se facilita la construcción de alianzas, permitiendo el acceso a herramientas de vigilancia tecnológica, estudios de mercado y propiedad intelectual, o se configuran fuentes de financiación de actividades que permiten acelerar las innovaciones, tales como el desarrollo de pruebas piloto (Aragón Amonarriz et al., 2017; Lai et al., 2012). Bonnin y O'Sullivan (2020) aducen que la principal causa del VM es la resistencia o poco interés del sector privado en invertir en tecnologías inmaduras. Para los autores, esto se debe, entre otras cosas, a la incertidumbre frente al 
hecho que dichas tecnologías si cumplan con los marcos normativos y regulatorios requeridos para su posible producción y comercialización.

Es preciso aclarar que este trabajo busca identificar los factores que participan en la formación del VM, en el marco de proyectos gestionados por entidades que cumplen el rol de agentes intermediarios de innovación de la ciudad de Medellín. De acuerdo con Ruiz Castañeda (2016), los intermediarios ayudan a cerrar brechas entre generadores y usuarios de las innovaciones tecnológicas. Se optó por estudiar los intermediarios asumiendo que, en tanto las innovaciones sean tecnológicas y hayan pasado previamente por fases de I+D, estos agentes deben enfrentar el VM. En este sentido, la pregunta problémica fue: ¿Qué factores inciden en la formación del estado de rezago que constituye el VM, y qué relaciones causales se dan entre ellos? La investigación arrojó información sobre la presencia de diversos factores candidatos a ser participantes en el VM, así como sobre las relaciones causales entre estos.

Este trabajo aporta a una línea de investigación tendiente a desarrollar modelos que den una explicación lo más acertada posible del comportamiento del VM que permita posteriormente explorar y definir políticas y enfoques de gestión de proyectos de I+D+i, buscando mejorar las probabilidades de superarlo. En esta primera fase se avanzó en identificar los principales factores a incluir en dichos modelos y sus relaciones causales.

En la primera parte del artículo se explica de forma detallada la metodología empleada; posteriormente se muestran los resultados obtenidos con la implementación de dicha metodología para cada uno de los factores analizados, así como una discusión en torno al significado de estos, interpretados a la luz del problema de investigación planteado. Finalmente se presentan las principales conclusiones del estudio, así como los referentes teóricos utilizados.

\section{METODOLOGÍA}

Se realizó un trabajo con enfoque cualitativo y de alcance descriptivo, a partir del análisis de factores presentes en nueve instituciones de educación superior (IES), lo que refuerza la posibilidad de contrastar datos (Martínez Carazo, 2006), comparando semejanzas o diferencias. El levantamiento de la información se concentró en la IES que tienen un rol protagónico en las actividades de CTi en Antioquia, específicamente en las universidades que componen el conglomerado denominado como G8 (Ruta N, 2013), y las IES Sapiencia, que hacen parte del subsistema de educación superior del Municipio de Medellín, de esta manera se buscó comprender qué sucede con dichos intermediarios.

La IES seleccionadas cubren alrededor del $72 \%$ de las capacidades de I+D+i registradas en Antioquia, y desde la perspectiva de los grupos de investigación categorizados en A, este valor pasa al $84 \%$ (Ministerio de Ciencia, Tecnología e Innovación, s.f.). La selección de esta muestra explica en gran parte lo que sucede en el ecosistema de I+D+i, al concentrar gran parte de las capacidades del departamento. En el proceso de análisis de información se incluyó documentación de los intermediarios, así como entrevistas a sus directivos. Dentro de cada IES, el tipo de intermediario considerado fueron las estructuras de transferencia o similares, las cuales son las encargadas de impulsar proyectos de I+D+i. 
La recolección de la información se realizó a partir de entrevistas a profundidad con los líderes de los procesos en cada IES, donde se indagó por los factores que inciden en el éxito o fracaso de los proyectos que son gestionados en sus respectivas entidades. Se partió de un listado de factores candidatos (Tabla 2), obtenidos y clasificados a partir de una revisión de literatura sobre barreras a la innovación (Negro et al., 2012; Van Lancker et al., 2016; Sandberg y Aarika, 2014; Jiménez, 2017). Los entrevistados consideraron los factores del listado inicial y aportaron algunos adicionales, basados en su experiencia y que fueron reportados como barreras para superar el VM en los proyectos de I+D+i gestionados por sus entidades. De igual forma se contrastó esta información con los resultados de un estudio previo (Jiménez, 2017) en donde se establecieron los que más influenciaban en la aparición del VM en Medellín.

Tras establecer los factores relevantes para cada entrevistado, estos elaboraron y aplicaron un diagrama causal simplificado, denominado círculo de conexión (Quaden et al., 2006), que sintetizó aquellos identificados para cada intermediario y sus relaciones causa-efecto. Esta información se transfirió a matrices de incidencia (Gil Lafuente y Barcellos de Paula, 2010; Gento et al., 2001), donde se realizó la homogeneizaron de los factores de cada IES. A continuación, se realizó una matriz global de incidencia de los mismos, donde se observó cuántas entidades seleccionaron las relaciones causaefecto detectadas en la matriz de incidencia.

Finalmente se elaboraron mapas radiales de las causas presentadas frente a los efectos de las columnas, tomando como referencia la gráfica de relaciones causa-efecto de factores que inciden en el VM. Esto se realizó por cada de uno de los factores analizados, representando de forma gráfica la relación existente e importancia de acuerdo con los entrevistados entre la causa y el efecto sobre cada factor. En la Figura 1 se presenta el resumen de la metodología.

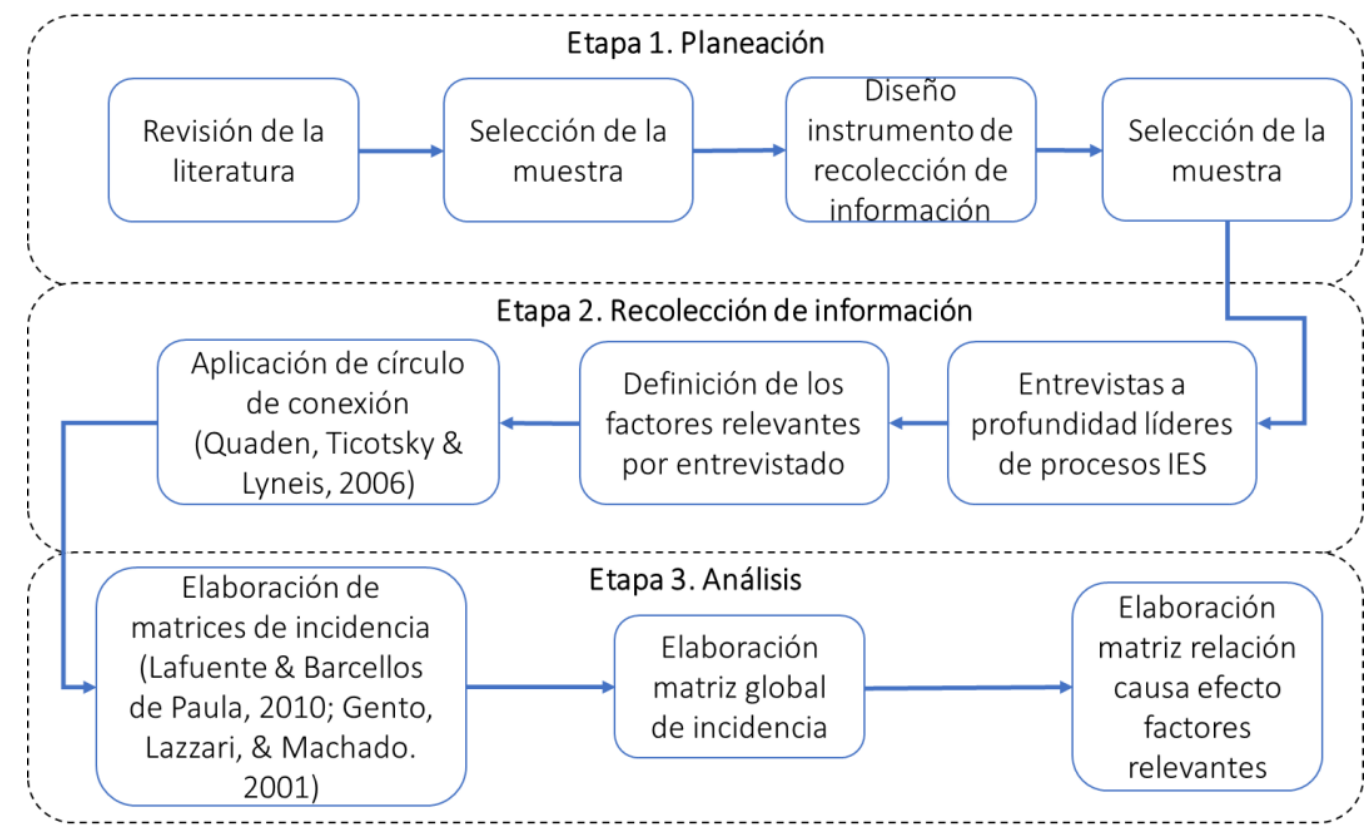

Figura 1. Metodología

Figure 1. Methodology

Fuente: elaboración propia. 


\section{RESULTADOS}

\section{Relaciones causa-efecto}

La información se recolecto a través de la aplicación de diagramas y a partir de entrevistas realizadas, donde los factores identificados en el VM (Tabla 2) se consolidaron en una matriz global que registró la cantidad de intermediarios de las IES que seleccionaron cada factor y cada relación causa-efecto entre ellos, los cuales, posteriormente, se consolidaron teniendo en cuenta su relevancia (Figura 2). Cada celda representa el valor dado por los entrevistados a partir de la valoración con una escala Likert de 1 a 6 , en la cual el intermediario indicó el efecto que tiene cada uno de los factores mostrados en las filas sobre aquellos establecidos en las columnas. De esta manera las relaciones causa-efecto con mayor peso entre todos las IES analizadas se presentan con tono verde oscuro (valor 6), las relaciones con peso igual o mayor a $50 \%$ se marcan con verde claro, las relaciones con peso entre 25 y $49 \%$ con amarillo, y las que tuvieron peso menor se marcan con color gris.

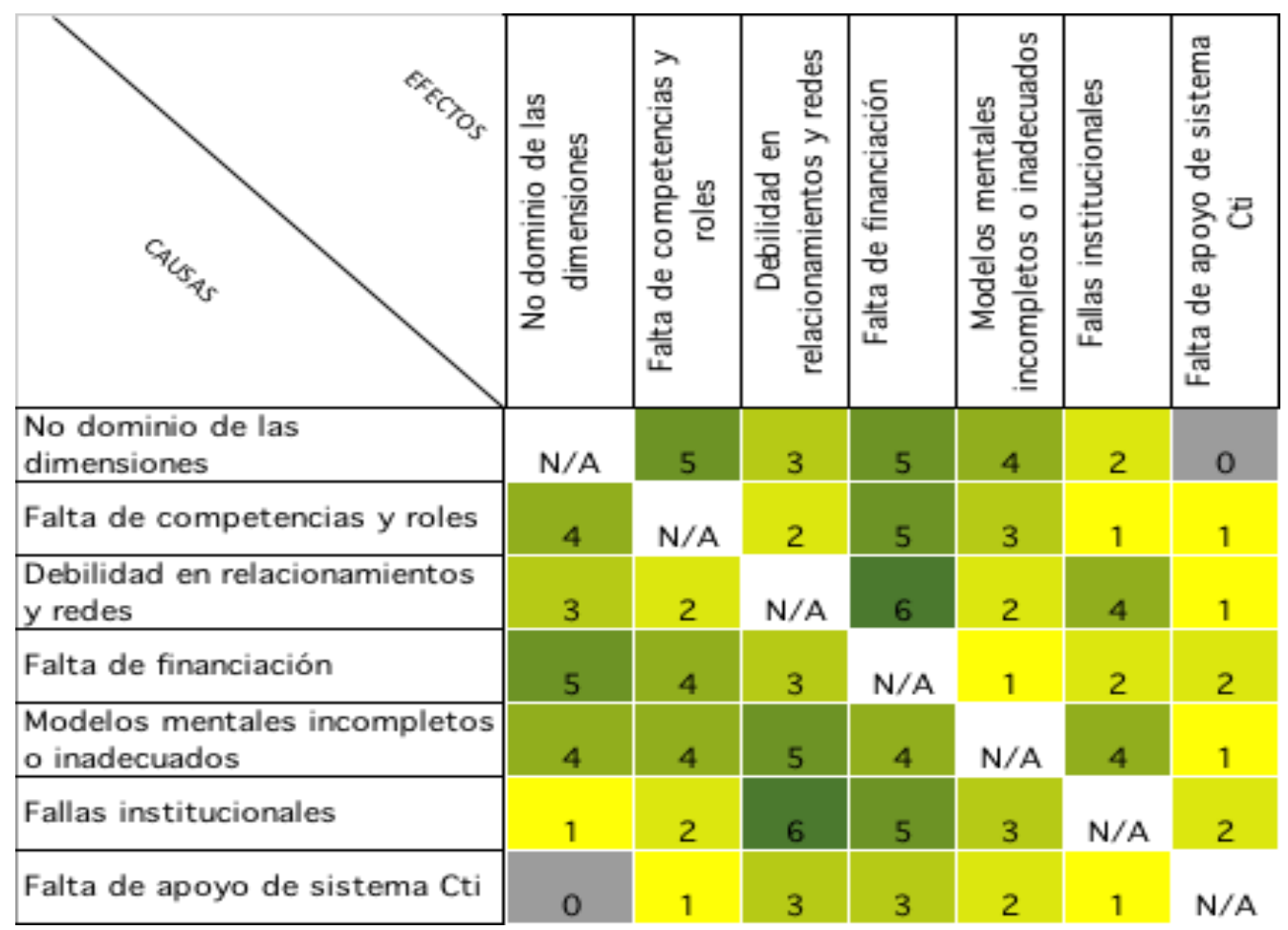

Figura 2. Relaciones causa-efecto de factores que inciden en el VM

Figure 2. Cause-effect relationships of the factors that influence the VoD Fuente: elaboración propia.

Las IES entrevistadas están de acuerdo que el factor que más incide en la aparición del VM es el No dominio de las dimensiones, seguido de la Falta de competencias. El tercer factor en importancia es la Debilidad en redes y relacionamientos. En cuarto lugar, se encuentra la Falta de financiación, este factor es uno de los más relevantes, encontrándose dificultades que van desde mayores requerimientos de financiar múltiples proyectos, lo que conduce a requerimientos de recursos mayores a su capacidad de financiación, así como la deficiencia de mecanismos que permitan fondear 
actividades de innovación que ya no corresponden a I+D Por su parte, los Modelos mentales ocupan el quinto lugar en orden de importancia como barrera para la innovación. En el sexto se halla las Fallas institucionales. Finalmente, la lista cierra con la Falta de apoyo del sistema CTi.

\section{No dominio de las dimensiones}

Se halló que existe relación causa-efecto entre el No dominio de las dimensiones (causa) y Falta de competencias y roles (efecto). Según los entrevistados, esto se explica en la medida que, aunque se cuente con talento humano dispuesto a resolver requerimientos de carácter científico, si no se integran personas que hagan labores no técnicas, tales como gestión de innovación y desarrollo del modelo de negocio, podría incidir en el desarrollo de aspectos clave para el éxito de proyectos de I+D+i. En este sentido se pudo evidenciar en las IES entrevistadas que la integración de equipos interdisciplinarios con asignación de tareas enfocadas en el acompañamiento a investigadores y sus proyectos con un enfoque desde la gestión de la innovación resultan un elemento clave para llevar a cabo procesos de transferencia de tecnología y conocimiento hacia la sociedad.

Frente al No dominio de las dimensiones (causa) y los Modelos mentales incompletos o inadecuados (efecto), se encontró que el desconocimiento de las dimensiones y la falta de visión en los aspectos no técnicos, involucrados en el desarrollo integral de proyectos de I+D hasta llegar a la explotación de las innovaciones, fomentan modelos mentales limitados o restrictivos, lo cual puede suceder incluso sin que la organización sea consciente de estas restricciones. Este desconocimiento es notable cuando un investigador de una universidad desarrolla modelos de negocios basados en el mérito tecnológico como característica suficiente para generar valor en el mercado. Usualmente dichos modelos de negocio no captan el interés de posibles inversionistas (Figura 3).

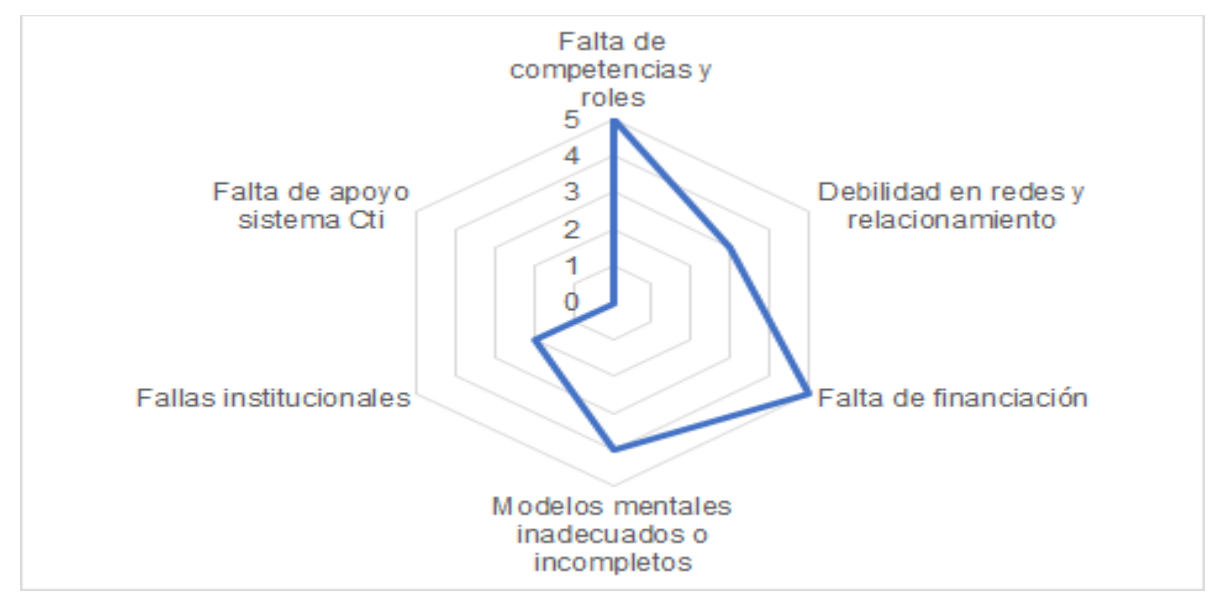

Figura 3. Mapa radial de ocurrencias del factor No dominio de las dimensiones

Figure 3. Radar chart of occurrences of the dimensional blindness factor Fuente: elaboración propia.

\section{Falta de competencias y roles}

En el comparativo de la Falta de competencia y roles (causa) con No dominio de las dimensiones, los entrevistados plantean que cuando se fortalecen las competencias del talento humano y la distribución de los roles dentro de los equipos innovadores, tiende a disminuirse la ceguera en las 
dimensiones, ya que un equipo de trabajo con múltiples competencias puede identificar y evaluar las dimensiones a tener en cuenta para encauzar el proyecto de I+D+i, más efectivamente hacia las etapas finales de innovación.

Además, se encontró una relación bidireccional entre competencias y trabajo en red; es decir, el primer factor es causa del segundo y viceversa. Los entrevistados explicaron que, si el equipo de trabajo de un proyecto no tiene competencias de gestión, o no se asume dicho rol, se dificulta adelantar actividades relacionadas con trabajo en red. De igual forma, un trabajo en red débil puede dificultar tanto el acceso como la formación de talento humano con las capacidades requeridas para el proyecto.

Frente a Falta de competencia (causa) y la Falta de financiación (efecto), los entrevistados indican que cuando los equipos se concentran en un área de conocimiento, o se especializan en los aspectos técnico-científicos, sin dominio de otros temas como la gestión, el conocimiento del mercado y sus necesidades, así como de la estrategia, es menor la receptividad de los eventuales financiadores, y el riesgo percibido aumenta (Figura 4).

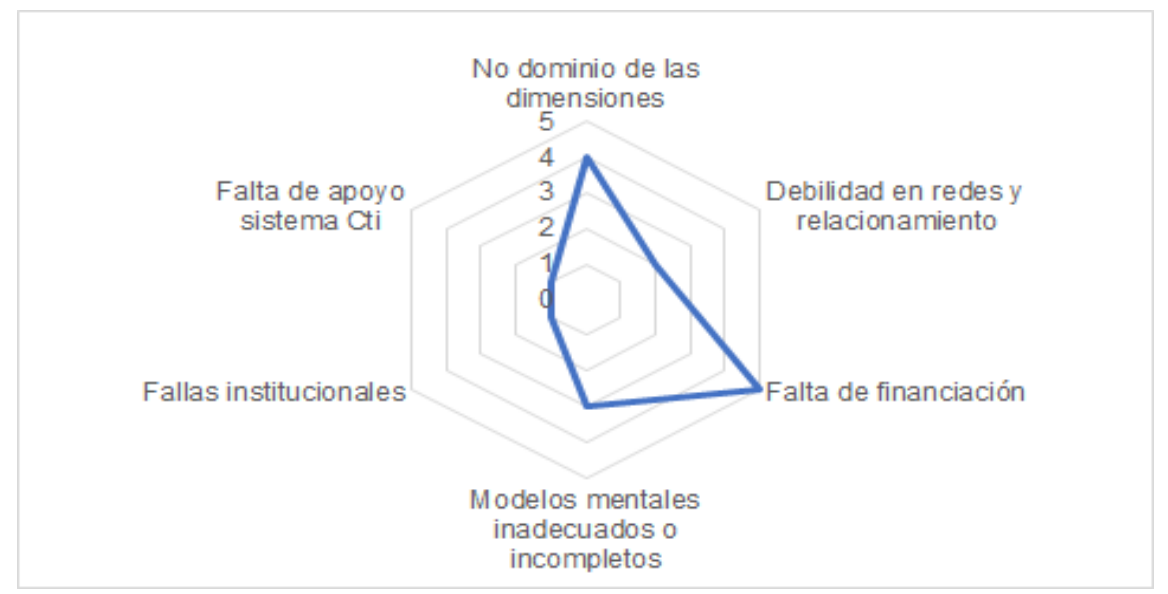

Figura 4. Mapa radial de ocurrencias del factor Falta de competencias y roles

Figure 4. Radar chart of occurrences of the lack of competences and roles factor Fuente: elaboración propia.

\section{Debilidad en redes y relacionamiento}

En la relación causa-efecto Debilidad en redes y relacionamiento con No dominio de las dimensiones, las IES analizadas indicaron que el relacionamiento con aliados aporta a una comprensión más integral, que ayuda a resolver la ceguera dimensional en virtud del aprendizaje por interacción.

La Debilidad en redes y relacionamiento y la Falta de financiación, es de especial atención para el trabajo de las IES, en este sentido si se demuestra a un inversionista el respaldo de aliados con experiencia y conocimiento, este podría acceder más fácilmente a invertir, mientras se perciben de mayor riesgo los proyectos de entidades individuales (Figura 5). 


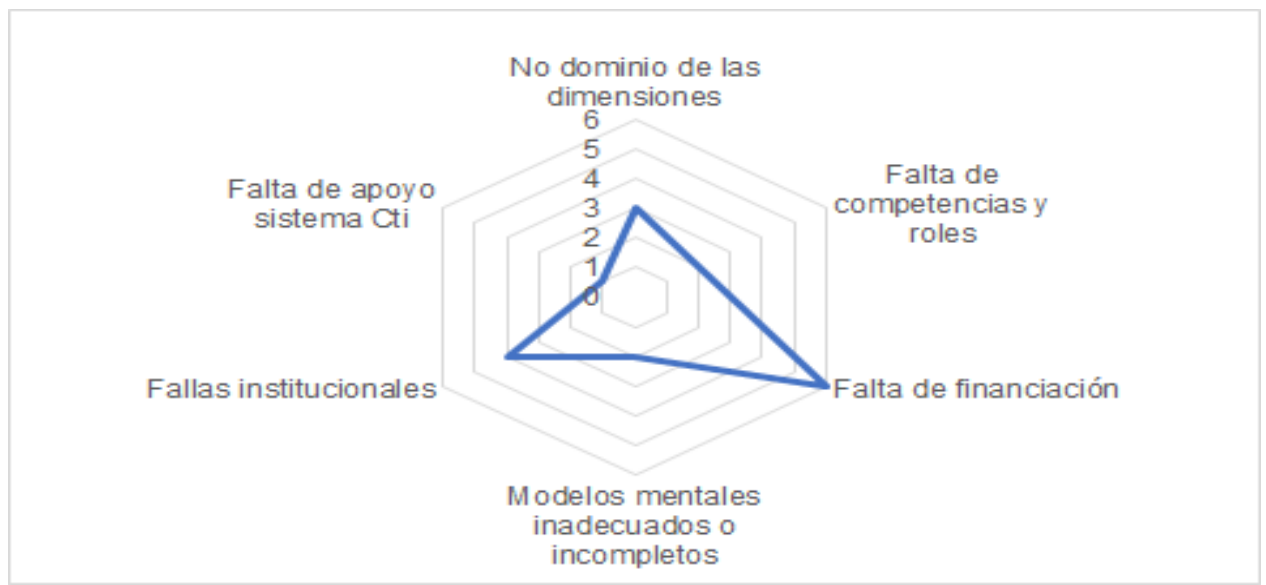

Figura 5. Mapa radial de ocurrencias del factor Debilidad en redes y relacionamiento Figure 5. Radar chart of occurrences of the weak networking and relationships factor Fuente: elaboración propia.

\section{Falta de financiación}

Existe una relación entre Falta de financiación (causa) con Falta de competencia y roles y el No dominio de las dimensiones; una debilidad en la baja financiación podría explicar la deficiencia en la generación de competencias al no poder contratar o asignar el talento humano necesario para el desarrollo de proyectos de I+D+i. Asimismo, por problemas de financiación, se pueden dejar de estudiar algunas dimensiones necesarias para entender el contexto del producto innovador y su posible éxito en el mercado. En algunos casos, estos estudios no técnicos no se presupuestaron desde el inicio del proyecto, y cuando se vuelve evidente la necesidad de efectuarlos, ya todos los recursos están asignados y la consecución de capital adicional puede ser una tarea compleja e infructuosa (Figura 6).

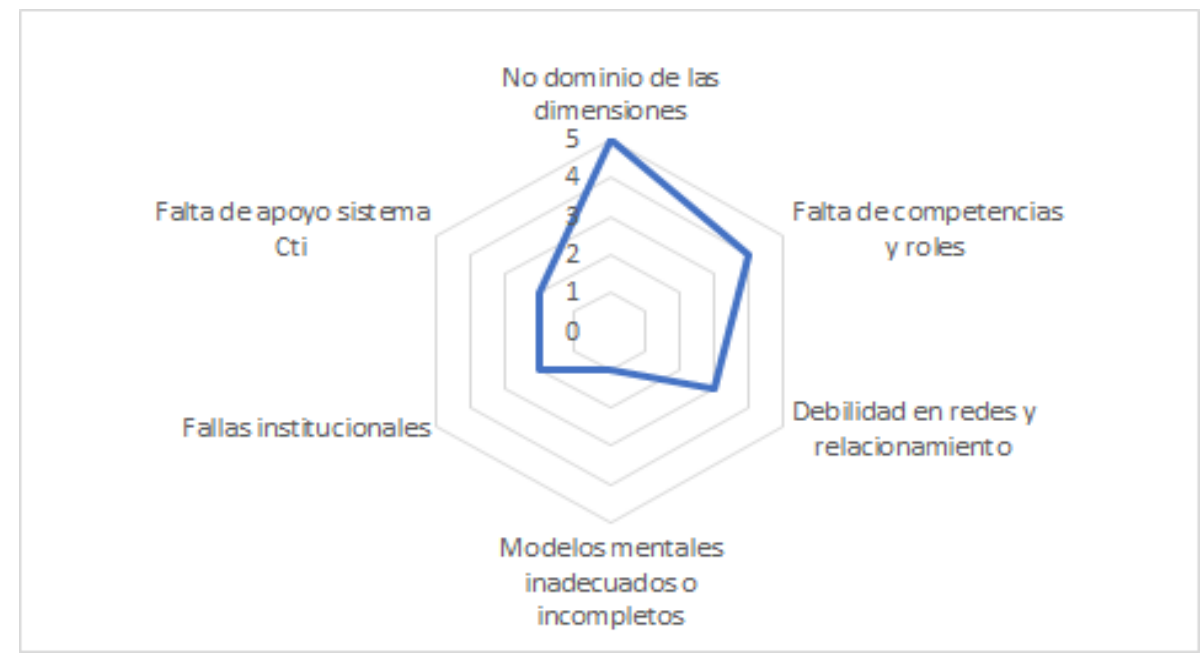

Figura 6. Mapa radial de ocurrencias del factor Falta de financiación

Figure 6. Radar chart of occurrences of the lack of funding factor Fuente: elaboración propia. 


\section{Modelos mentales incompletos o inadecuados}

Se encontró una relación entre Modelos mentales incompletos o inadecuados y Falta de competencias y roles. En este sentido, cuando las IES tienen una mentalidad restrictiva, o los equipos de proyecto tienen modelos mentales inmaduros, no alcanzan a prever todos los problemas que se deben abordar y las capacidades que deben dominar para desarrollar proyectos de I+D+i, ni se logran definir completa y adecuadamente los roles que deben ser cumplidos por el talento humano. En el caso de las universidades, a algunos investigadores se les exige que tengan roles de emprendedores o negociadores de las innovaciones, y en ocasiones, no tienen capacidades, disposición o el interés de hacerlo (Figura 7).

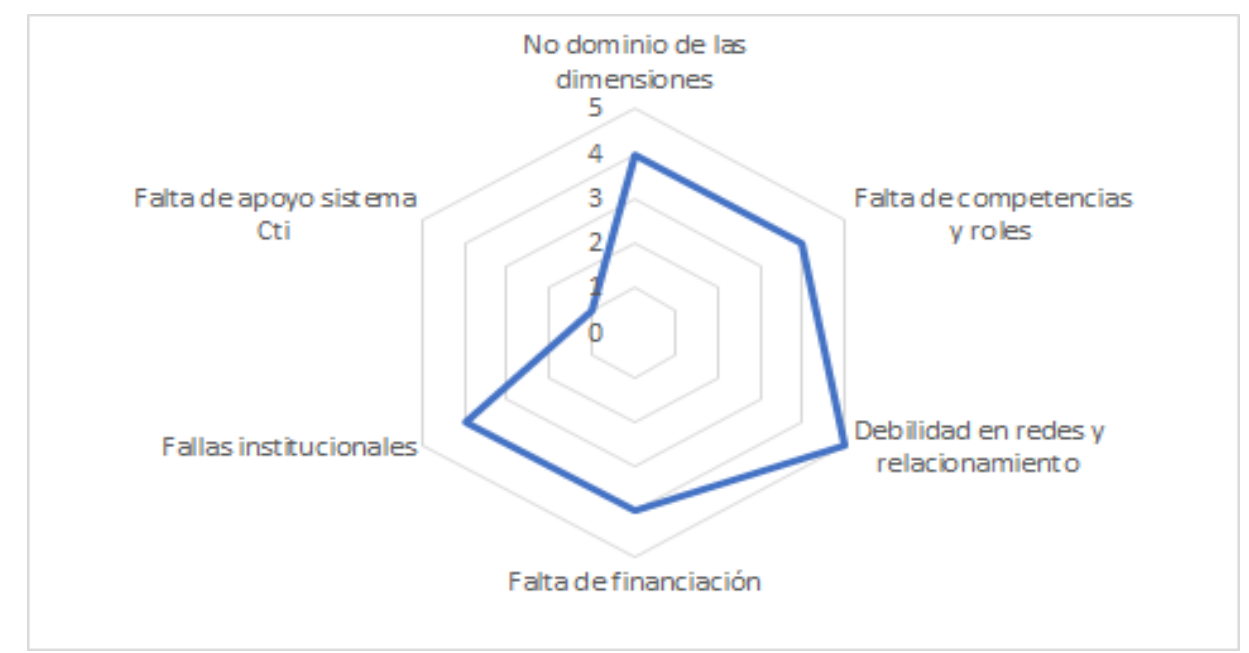

Figura 7. Mapa radial de ocurrencias de Modelos mentales incompletos o inadecuados

Figure 7. Radar chart of occurrences of the incomplete or inadequate mental models factor Fuente: elaboración propia.

\section{Fallas institucionales}

En el análisis de las Fallas institucionales y la Debilidad en redes y relacionamiento, se encontraron dificultades para establecer alianzas debido al conjunto de reglas existentes, normativas internas, protocolos y procedimientos en las universidades para gestionar alianzas con terceros; dichos fenómenos se presentan con especial énfasis en las IES de carácter públicas. Esta dificultad retrasa las actividades colaborativas, e incluso se reportan eventos donde los aliados desisten tras encontrar excesivas las condiciones a cumplir y los tiempos de trámite involucrados (Figura 8).

\section{Falta de apoyo del sistema CTi}

Este es un factor favorable pero no determinante, ya que es posible encontrar proyectos exitosos que no recibieron ningún tipo de apoyo del sistema CTi, pero por otro lado también se tienen proyectos fallidos que lo recibieron. El factor se enfoca en la interacción de actores intermediaros del sistema de apoyo CTi en función de proyectos de I+D+i, de forma que se generen actividades de apoyo para las entidades que se encuentran desarrollando proyectos innovadores. 
Este apoyo puede ser de dos tipos: el aporte de capacidades a un proyecto I+D+i y, de esta manera, adelantar algunas actividades que hacen parte de la ejecución del proyecto, tales como vigilancia tecnológica, formulación de los modelos de negocio, estudios de mercado, propiedad intelectual, entre otros. De esta manera, el apoyo del sistema CTi se traduce en el fortalecimiento de alianzas que prestan capacidades a los proyectos innovadores. Por otro lado, se tiene la entrega de recursos financieros para el desarrollo y ejecución de pruebas piloto, desarrollo de producto mínimo viable y la contratación de estudios como planes de comercialización o asesorías en temas de modelos financieros, el cual se manifiesta en forma de aliados o ayudas técnicas mediante la prestación de servicios que ayudan a resolver determinadas dimensiones del proyecto o financiación de actividades pendientes (Figura 9).

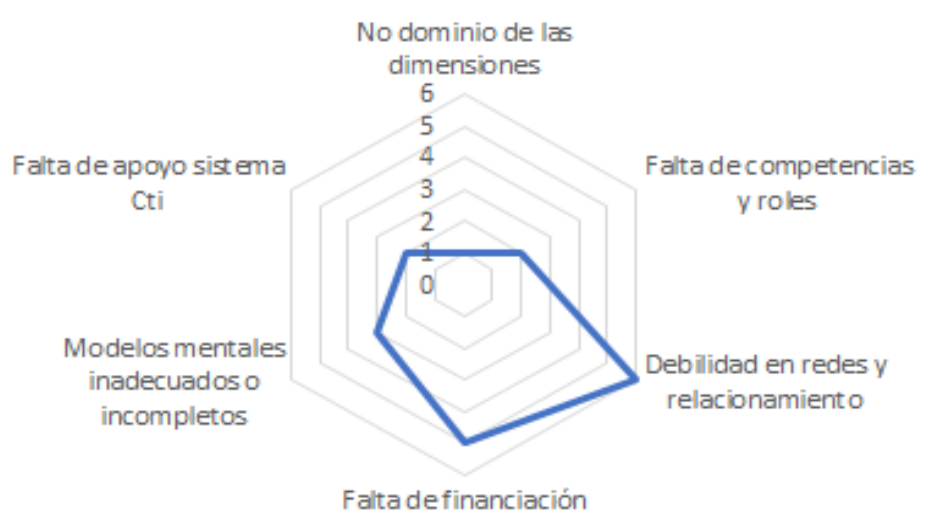

Figura 8. Mapa radial de ocurrencias del factor Fallas institucionales

Figure 8. Radar chart of occurrences of the institutional failures factor Fuente: elaboración propia.

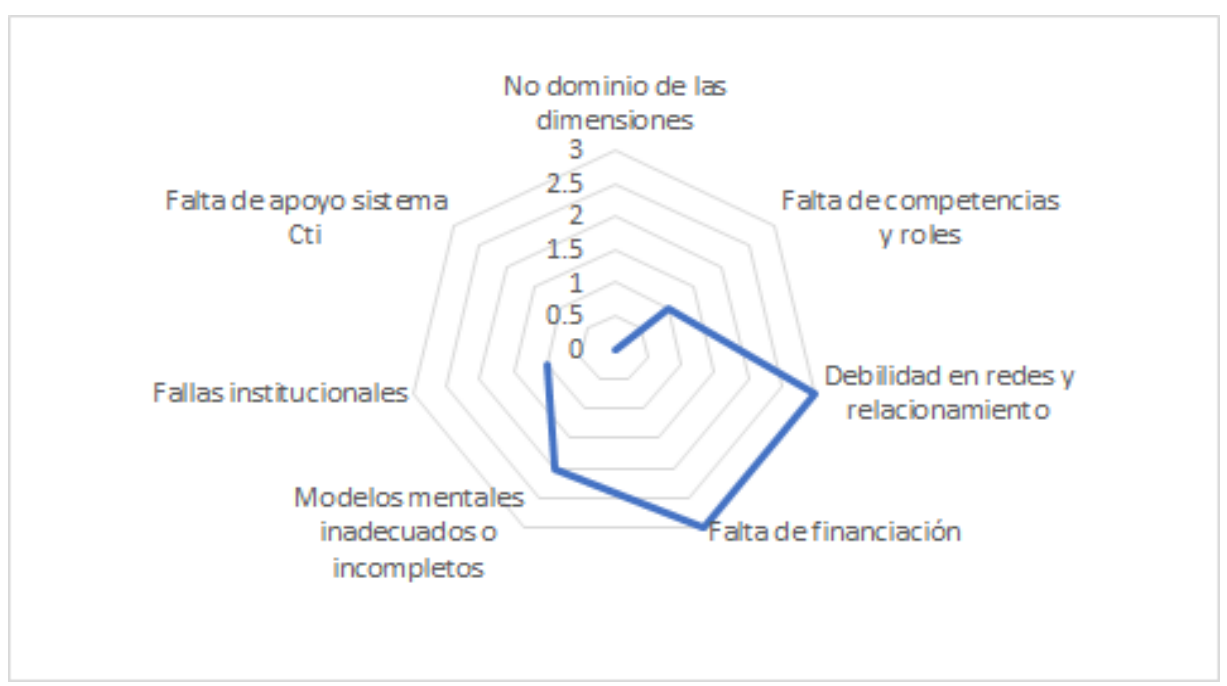

Figura 9. Mapa radial de ocurrencias del factor Falta de apoyo del sistema CTi Figure 9. Radar chart of occurrences of the lack of support from the STI system factor Fuente: elaboración propia. 


\section{DISCUSIÓN}

Si bien este trabajo llevó a reconocer 33 factores generadores del VM, fue posible identificar un subconjunto de aparición reiterada, encabezado por las competencias, conocimiento de las dimensiones, trabajo en red, financiación, instituciones y modelos mentales. También se reconocieron relaciones causales entre estos, y en particular una fuerte relación entre el primero (competencias y roles) y los otros del subconjunto anterior, con excepción de la normatividad. Autores como Flores-Aguero et al. (2021) añaden a esta discusión la importancia de la prospectiva tecnológica como herramienta para mejorar las capacidades centrales de los centros de investigación; los investigadores concluyen que efectivamente la prospectiva tecnológica se debe integrar como una capacidad central de las organizaciones de manera que esta le permita generar ventajas competitivas en el mercado.

A partir de un estudio de caso en una organización del sector salud, Vega-González y Vega-Salinas (2020) plantean en su investigación la necesidad de mejorar la vinculación y la gestión de proyectos tecnológicos entre instituciones públicas y privadas; dentro de las conclusiones a las que llegan los autores se tiene el hecho de la importancia de la construcción de redes de innovación en el ámbito nacional e internacional con el objetivo de complementar las capacidades de gestión de recursos y proyectos en I+D, accediendo a distintas fuentes de financiación. De igual forma mencionan el hecho de acercar la oferta tecnológica de las distintas instituciones que componen el sistema de I+D con el público en general, de manera que los resultados que se obtengan sean más pertinentes con el contexto.

Para Rojas-Arenas et al. (2017), una adecuada interacción del sistema de CTi en cualquier región especifica es fundamental para alcanzar niveles de desarrollo económico local, fundamentados en procesos de cambio estructural mediados por la innovación.

La presente investigación se centra en la idea que, para comprender mejor el VM, es relevante un enfoque sistémico y complejo; esto en la medida en que se consideren diversos tipos de factores y sus relaciones causales, lo cual es fundamental cuando se habla de un sistema complejo, siendo en este, precisamente, donde radica la complejidad en las variables que lo componen y las interacciones que se dan entre estas (Morin, 1995 citado por Rojas Arenas, 2018).

Así las cosas, los resultados ayudan a soportar la idea planteada en el párrafo anterior, al mostrar un conjunto de factores de diversa índole (competencias, conocimiento de las dimensiones, trabajo en red, financiación, entre otros), en los cuales se reconocen interrelaciones. Estos hallazgos dan pie a formular una explicación alternativa a los modelos previos que se han centrado en un factor, o en unos cuantos afines entre sí.

Buena parte de la literatura considera la financiación como factor dominante del VM (Frank et al., 1996; Auerswald y Branscomb, 2003; Beard et al., 2009; Munari et al., 2018). Al analizar los resultados, se encontró que, en las entidades revisadas, la financiación es un factor importante, aunque no es el único, ni el de mayor peso. También se identificó que el acceso a la financiación guarda una relación con el trabajo en red, las competencias y roles del equipo humano y con la ceguera dimensional. Comprender esta estructura de relaciones será útil para elaborar modelos 
sistémicos del VM que trascienden la interpretación financiera y se muestre la disposición de recursos como parte de una interacción sistémica multifactorial.

Las competencias aparecen como factor destacado, aunque no exclusivo. Esto da pie a trabajos futuros que profundicen en las características del equipo humano participante en las innovaciones tecnológicas, buscando una mayor comprensión de los rasgos personales y de las responsabilidades a asumir. Para ello puede aprovecharse un modelo esbozado por Markham (2010) y enriquecido por Markham et al. (2016) que relaciona la presencia de tres roles: campeones, patrocinadores y guardianes, con la ejecución de gestiones necesarias para que los proyectos superen el VM.

\section{CONCLUSIONES}

Una vez concluida la investigación se observa cómo el análisis de los múltiples factores que intervienen en el VM y su relación pueden brindar una explicación, al menos parcial, de cómo estos generan este fenómeno, lo cual es un resultado importante teniendo en cuenta que una vez se conocen las causas y efectos de un objeto de estudio se pueden establecer rutas de acción más precisas para realizar una mejor gestión de los proyectos de I+D+i en las organizaciones. Además, se muestra la importancia de algunos factores que generalmente no son tenidos en cuenta (como los modelos mentales o la falta de claridad en roles y competencias), lo que, a su vez, presupone ir más allá del paradigma reinante frente al tema de la financiación como factor más determinante del fracaso o éxito de los proyectos de I+D.

Se considera que una de las fortalezas de la presente investigación radica en el hecho de tomar como objeto de estudio un fenómeno poco analizado en el medio, lo cual permite abrir una línea de investigación en esta temática, considerándose de vital importancia para el éxito de los procesos locales de innovación; ahora bien, aunque inicialmente el alcance de esta estaría limitado para la ciudad de Medellín, es claro cómo a partir de la metodología utilizada, y complementando la misma, se pueden formular otros proyectos con un mayor alcance y que, por tanto, pueda nutrirse el ecosistema de innovación.

Dado lo anterior, los resultados de este trabajo tienen potencial y pueden servir de insumo para mejorar la gestión por parte de intermediarios, y en general la gestión de proyectos de I+D+i. Los hallazgos del estudio son insumos para un modelo sistémico que permita entender mejor el VM y simular políticas y estrategias de gestión que mejoren las probabilidades de éxito de las innovaciones. En tanto, en la medida que dicho modelo contribuya a reducir los tiempos muertos de los proyectos de I+D+i y a incrementar el éxito de los proyectos de innovación, se podrá considerar que se cumplió con el cometido.

\section{CONFLICTOS DE INTERÉS}

Los autores declaran que no presentan conflictos de interés financiero, profesional o personal que pueda influir de forma inapropiada en los resultados obtenidos o las interpretaciones propuestas. 


\section{CONTRIBUCIÓN DE AUTORES}

Para el desarrollo de este proyecto todos los autores han realizado una contribución significativa especificada a continuación:

Elizabeth Jiménez-Medina: el trabajo de maestría de la autora sirvió como referencia para esta publicación, de hecho, con base en el mismo se construyó el marco y teórico. Así mismo, actualizó referentes sobre la temática, apoyó la construcción del apartado metodológico y el análisis de los resultados. Junto con el investigador Rene Yepes, planteó la problemática de la cual se deriva la investigación.

René Yepes-Callejas: apoyó la construcción del marco teórico; construyo en conjunto con los otros autores el apartado metodológico y se encargó de una gran parte del análisis de los resultados. Así mismo, aportó en el capítulo de conclusiones, y dio una base de referentes teóricos importantes para la investigación. Junto con la Investigadora Elizabeth Jiménez, estableció los criterios generales para la investigación.

Jim Giraldo-Builes: apoyó la construcción del apartado teórico y metodológico, así como el análisis de los resultados y las conclusiones Fue uno de los encargados de hacer las correcciones en las diferentes etapas de evaluación del artículo. Elaboró todos los gráficos requeridos en el texto, y aportó en el análisis y descripción de estos. Se encargó además del análisis de diferentes fuentes bibliográficas para actualizar los referentes teóricos.

Iván Darío Rojas-Arenas: apoyó la construcción de los apartados teórico, metodológico, análisis de resultados y conclusiones. Realizó la revisión general del texto para la corrección de texto y se encargó junto al investigador Jim Giraldo de realizar las respectivas correcciones derivadas del proceso editorial, también se encargó de hacer el resumen, abstract y de ajustar el articulo a los requerimientos de la revista.

\section{REFERENCIAS}

Abereijo, I. O. (2015). Transversing the «valley of death»: Understanding the determinants to commercialisation of research outputs in Nigeria. African Journal of Economic and Management Studies, v. 6, n. 1, 90-106. https://doi.org/10.1108/AJEMS-10-2012-0066

Alunni, A. (2020). La importancia de la inversión de riesgo corporativo para superar el valle de la muerte en la transferencia tecnológica universitaria. Revista de Fomento Social, n. 296, 139156. https://doi.org/10.32418/rfs.2020.296.3311

Andronchev, I. K., Garanin, M. A., Vladimirovna, V., Bolgova, V. V. A., Bulatov, A. A. (2020). Improving the efficiency of innovation development and implementation. The IIOAB Journal, v. 11, $\mathrm{n}$. S3, 52-56. URL 
Aragón Amonarriz, C., Iturrioz, C., Narvaiza, L., Parrilli, M. D. (2017). The role of social capital in regional innovation systems: Creative social capital and its institutionalization process. Papers in Regional Science, v. 98, n. 1, 35-51. https://doi.org/10.1111/pirs.12329

Auerswald, P. E., Branscomb, L. M. (2003). Valleys of Death and Darwinian Seas: Financing the Invention to Innovation Transition in the United State. Journal of Technology Transfer, v. 28, 227-239. https://doi.org/10.1023/A:1024980525678

Barron, E., Amorós, J. E. (2020). Bridging the Valley of Death: Lessons from an Entrepreneurial Program. International Journal of Entrepreneurship, v. 24, n. 1. URL

Beard, T. R., Ford, G. S., Koutsky., T. M., Spiwak, L. J. (2009). A Valley of Death in the innovation sequence: an economic investigation. Research Evaluation, v. 18, n. 5, 343-356. https://doi.org/10.3152/095820209X481057

Bonnin Roca, J., O'Sullivan, E. (2020). The role of regulators in mitigating uncertainty within the Valley of Death. Technovation, 102157. https://doi.org/10.1016/i.technovation.2020.102157

Botha, M., Morallane, H. (2019). Cognitive adaptability and narrow personality traits: Proposing a person-entrepreneurship fit for established entrepreneurs in South Africa. South African Journal of Business Management, v. 50, n. 1, 1-10. https://doi.org/10.4102/sajbm.v50i1.1323

Daldrup, V., Krahl, O., Bürger, R. (2020). Is Crowdfunding Suitable for Financing German Public Research Organization (PRO) Projects? En Moritz A., Block J., Golla S., Werner A. (eds), Contemporary Developments in Entrepreneurial Finance (309-333). Springer. https://doi.org/10.1007/978-3-030-17612-9 12

de Frutos Belizón, J., Martín Alcázar, F., Sánchez Gardey, G. (2019). Gestionando el "Valle de la muerte" entre académicos y profesionales en el campo del management: Una evidencia empírica. Cuadernos de Gestión, v. 19, n. 1, 87-118. https://doi.org/10.5295/cdg.170745jf

Flores-Aguero, F., Vivanco-Vargas, M., Méndez-Palacios, J. J., Bermudez-Peña, C. P. (2021). La prospectiva tecnológica desde la perspectiva de las capacidades centrales en centros de investigación. Revista CEA, v. 7, n. 13, e-1628. https://doi.org/10.22430/24223182.1628

Frank, C., Sink, C., Mynatt, L., Rogers, R., Rappazzo, A. (1996) Surviving the «Valley of Death»: A comparative analysis. Journal of Technology Transfer, v. 21, 61-69. https://doi.org/10.1007/BF02220308

García Núñez, J. A., Varón Cárdenas, D., Barrera Hernández, J. C. (2019). Convirtiendo una planta de beneficio en una biorrefinería: paso de tecnologías por el valle de la muerte. Revista Palmas, v. 40, n. Especial T, 76-103. URL

García, R., Calantone, R. (2003). A critical look at technological innovation typology and innovativeness terminology: a literature review. Journal of Product Innovation Management, v. 19, n. 2, 110-132. https://doi.org/10.1111/1540-5885.1920110 
Valle de la muerte: factores que dificultan el éxito de innovaciones tecnológicas

Gento, A., Lazzari, L., Machado, E. (2001). Reflexiones acerca de las matrices de incidencia y la recuperación de efectos olvidados. Cuadernos del CIMBAGE, n. 4, 11-27. URL

Gil Lafuente, A. M., Barcellos de Paula, L. (2010). Una aplicación de la metodología de los efectos olvidados: los factores que contribuyen al crecimiento sostenible de la empresa. Cuadernos del CIMBAGE, n. 12, 23-34. URL

Gimeno, J. Á., Llera, E., Scarpellini, S. (2018). Investment determinants in self-consumption facilities: characterization and qualitative analysis in Spain. Energies, v. 11, n. 8, 2178. https://doi.org/10.3390/en11082178

González Fernández, S., Kubus, R., Pérez-Iñigo, J. M. (2019). Innovation ecosystems in the EU: Policy evolution and horizon Europe proposal case study (the Actors' perspective). Sustainability, v. 11, n. 17, 4735. https://doi.org/10.3390/su11174735

Gou, J., Li, J., Ruan, P. (2013). Strategic Research of the Crossing of the "Death Valley» in NewlyEmerging Industry. Atlantis Press, 719-725. URL

Gubitta, P., Tognazzo, A., Destro, F. (2016). Signaling in academic ventures: the role of technology transfer offices and university funds. The Journal of Technology Transfer, v. 41, 368-393. https://doi.org/10.1007/s10961-015-9398-7

Hsu, P., Tian, X., Xu, Y. (2014). Financial development and innovation: Cross-country evidence. Journal of Financial Economics, v. 112, n. 1, 116-135. https://doi.org/10.1016/j.jfineco.2013.12.002

Ivanova, D., Haradinova, A., Vasileva, E. (2019). Eco-Innovations in Bulgarian Companies with ProEnvironmental Policy. Quality-access to success, v. 20, n. 168, 107-112. URL

Jiménez, E. (2017). Factores que inciden en el Valle de la Muerte en proyectos de 1+D+i en Medellín y su Área Metropolitana [Tesis de maestría]. Universidad Pontificia Bolivariana.

Lai, X., Ye, Z., Xu, Z., Husar Holmes, M., Henry Lambright, W. (2012). Carbon capture and sequestration (CCS) technological innovation system in China: Structure, function evaluation and policy implication. Energy Policy, v. 50, 635-646.

https://doi.org/10.1016/i.enpol.2012.08.004

Lee-Kelley, L., Blackman, D. (2005). In addition to shared goals: the impact of mental models on team innovation and learning. International journal of innovation and Learning, v. 2, n. 1, 11-25. https://doi.org/10.1504/IJIL.2005.006080

Lettner, M., Hesser, F., Hedeler, B., Schwarzbauer, P., Stern, T. (2020). Barriers and incentives for the use of lignin-based resins: Results of a comparative importance performance analysis. Journal of Cleaner Production, v. 256, 120520. https://doi.org/10.1016/i.jclepro.2020.120520 
Liening, A., Geiger, J.-M., Kriedel, R. (2018). Bridging the Gap Between Invention and Innovation: The Role of University-Based Start-Up Programs and Private Cooperation. En Presse, A. y Terzidis, O. (ed.), Technology Entrepreneurship (241-260). https://doi.org/10.1007/978-3-319-73509-2 13

Markham, S. K. (2016). Moving technologies from lab to market. Research-technology Management, v. 45, n. 6, 31-42. https://doi.org/10.1080/08956308.2002.11671531

Markham, S. K., Mugge, P. (2015). Traversing the Valley of Death. A practical guide for innovation leader.

Markham, S. K., Ward, S. J., Aiman-Smith, L., Kingon, A. I. (2010). The Valley of Death as Context for Role Theory in Product Innovation. Journal of Product Innovation Management, v. 27, n. 3, 402-417. https://doi.org/10.1111/j.1540-5885.2010.00724.x

Martínez Carazo, P. C. (2006). El método de estudio de caso: estrategia metodológica de la investigación científica. Pensamiento \& Gestión, n. 20, 165-193. URL

Merrifield, D. B. (1995). Obsolescence of core competencies versus corporate renewal. Technology Management, v. 2, n. 2, 78-83. URL

Meyer, A. D., Aten, K., Krause, A. J., Metzger, M. L., Holloway, S. S. (2011). Creating a university technology commercialisation programme: confronting conflicts between learning, discovery and commercialisation goals. International Journal of Entrepreneurship and Innovation Management, v. 13, n. 2, 179-198. https://doi.org/10.1504/IJEIM.2011.038858

Ministerio de Ciencia, Tecnología e Innovación. (s.f.). La ciencia en cifras. URL

Munari, F., Sobrero, M., Toschi, L. (2018). The university as a venture capitalist? Gap funding instruments for technology transfer. Technological Forecasting and Social Change, v. 127, 7084. https://doi.org/10.1016/i.techfore.2017.07.024

Muso, R., Echecopar, G. (2012), El Valle de la Muerte: cómo superar la partida al emprender. Amazon.

Negro, S. O., Alkemade, F., Hekkert, M. P. (2012). Why does renewable energy diffuse so slowly? A review of innovation system problems. Renewable and Sustainable Energy Reviews, v. 16, n. 6, 3836-3846. https://doi.org/10.1016/j.rser.2012.03.043

Nemet, G. F., Zipperer, V., Kraus, M. (2018). The valley of death, the technology pork barrel, and public support for large demonstration projects. Energy Policy, v. 119, 154-167. https://doi.org/10.1016/i.enpol.2018.04.008

Padyab, A., Habibipour, A., Rizk, A., Ståhlbröst, A. (2020). Adoption barriers of loT in large scale pilots. Information, v. 11, n. 1, 23. https://doi.org/10.3390/info11010023 
Valle de la muerte: factores que dificultan el éxito de innovaciones tecnológicas

Quaden, R., Ticotsky, A., Lyneis, D., (2006). The Shape of Change: Stocks and Flows. Creative learning exchange.

Raven, R. P. J. M., Geels, F. W. (2010). Socio-cognitive evolution in niche development: Comparative analysis of biogas development in Denmark and the Netherlands (1973-2004). Technovation, v. 30, n. 2, 87-99. https://doi.org/10.1016/j.technovation.2009.08.006

Rojas Arenas, I. D. (2018). Del desarrollo económico al desarrollo económico endógeno: una mirada desde el pensamiento complejo. Revista CINTEX, v. 23, n. 1, 12-23.

https://doi.org/10.33131/24222208.308

Rojas-Arenas, I. D., Cardona-Quintero, J. A., Gallego-Alzate, J. B. (2017). Estrategias de desarrollo económico local para el municipio de Girardota. Revista CEA, v. 3, n. 6, 29-45. https://doi.org/10.22430/24223182.770

Rothaermel, F. T., Deeds, D. L. (2006). Alliance type, alliance experience and alliance management capability in high-technology ventures. Journal of Business Venturing, v. 21, n. 4, 429-460. https://doi.org/10.1016/i.jbusvent.2005.02.006

Ruiz Castañeda, W. L. (2016). Análisis del impacto de los intermediarios en los sistemas de innovación: Una propuesta desde el modelado basado en agentes. [Tesis de doctorado]. Universidad Nacional de Colombia. https://repositorio.unal.edu.co/handle/unal/56636

Ruta N. (2013). El G8: Un acuerdo de voluntades. $\underline{\text { URL }}$

Sandberg, B., Aarikka-Stenroos, L. (2014). What makes it so difficult? A systematic review on barriers to radical innovation. Industrial Marketing Management, v. 43, n. 8, 1293-1305. https://doi.org/10.1016/j.indmarman.2014.08.003

Schoonmaker, M., Carayannis, E., Rau, P. (2013). The role of marketing activities in the fuzzy front end of innovation: a study of the biotech industry. Journal of Technology Transfer, v. 38, n. 6, 850-872. https://doi.org/10.1007/s10961-012-9296-1

Surroca, J., Prior, D., Tribó Giné, J. A. (2014). Using panel data dea to measure CEOs' focus of attention: An application to the study of cognitive group membership and performance. Strategic Management Journal, v. 37, n. 2, 370-388. https://doi.org/10.1002/smj.2350

Tassey, G. (2014). Competing in Advanced Manufacturing: The Need for Improved Growth Models and Policies. Journal of Economic Perspectives, v. 28, n. 1, 27-48. https://doi.org/10.1257/jep.28.1.27

Van Lancker, J., Mondelaers, K., Wauters, E., Huylenbroeck, G. (2016). The Organizational Innovation System: A systemic framework for radical innovation at the organizational level. Technovation, v. 52-53, 40-50. https://doi.org/10.1016/i.technovation.2015.11.008 
Vaníčková, R., Szczepańska-Woszczyna, K. (2020). Innovation of business and marketing plan of growth strategy and competitive advantage in exhibition industry. Polish Journal of Management Studies, v. 21, n. 2, 425-445. https://doi.org/10.17512/pjms.2020.21.2.30

Vega-González, L. R., Vega-Salinas, R. M. (2020). Plan para desarrollar redes de vinculación y gestión en Instituciones de I\&D en Salud: estudio de caso. Revista CEA, v. 6, n. 12, 105-127. https://doi.org/10.22430/24223182.1504

Vink, J., Edvardsson, B., Wetter-Edman, K., Tronvoll, B. (2019). Reshaping mental models-enabling innovation through service design. Journal of Service Management, v. 30, n. 1, 75-104. https://doi.org/10.1108/JOSM-08-2017-0186

Weiss, C., Bonvillian, W. B. (2013). Legacy sectors: barriers to global innovation in agriculture and energy. Technology Analysis \& Strategic Management, v. 25, n. 10, 1189-1208. https://doi.org/10.1080/09537325.2013.843658 